\title{
Stochastic fractional perturbed control systems with fractional Brownian motion and Sobolev stochastic non local conditions
}

\author{
Kerboua Mourad $^{1}$ • Ellaggoune Fateh $^{1}$. \\ Dumitru Baleanu ${ }^{2,3}$
}

Received: 5 March 2017 / Accepted: 3 October 2017 / Published online: 20 October 2017

(C) The Author(s) 2017. This article is an open access publication

\begin{abstract}
This paper investigates the approximate controllability for Sobolev type stochastic perturbed control systems of fractional order with fractional Brownian motion and Sobolev fractional stochastic nonlocal conditions in a Hilbert space, A new set of sufficient conditions are established by using semigroup theory, fractional calculus, stochastic integrals for fractional Brownian motion, Banach's fixed point theorem. The results are obtained under the assumption that the associated linear system is approximately controllable. Finally, an example is also given to illustrate the obtained theory.
\end{abstract}

Keywords Approximate controllability $\cdot$ Perturbed control systems · Fractional stochastic system · Fixed point technique $\cdot$ Stochastic nonlocal condition · Fractional Brownian motion

Mathematics Subject Classification 93B05 · 26A33 - 46E39 - 60H15 - 60G22

\section{Introduction}

The focus of this investigation is the approximate controllability problem for a class of Sobolev type stochastic perturbed control systems of fractional order with fractional sobolev stochastic nonlocal conditions and fractional Brownian motion of the form

Kerboua Mourad

kerbouamourad@gmail.com

Ellaggoune Fateh

fellaggoune@gmail.com

Dumitru Baleanu

dumitru@cankaya.edu.tr

1 Department of Mathematics, Guelma University, 24000 Guelma, Algeria

2 Department of Mathematics and Computer Sciences, Cankaya University, 06530 Ankara, Turkey

3 Institute of Space Sciences, Magurele-Bucharest, Romania 


$$
\begin{aligned}
{ }^{C} D_{t}^{q}[L x(t)]= & (M+\Delta M) x(t)+B u(t)+f(t, x(t)) \\
& +g_{1}(t) \frac{d B_{1}^{H}(t)}{d t}, \quad t \in J=[0, T], \\
{ }^{L} D_{t}^{1-q}[V x(0)]= & g_{2}(t) \frac{d B_{2}^{H}(t)}{d t},
\end{aligned}
$$

where the state $x(\cdot)$ takes values in a separable real Hilbert space $X$ with inner product $\langle\cdot, \cdot\rangle_{X}$ and norm $\|\cdot\|_{X}$. Here $0<q \leq 1,{ }^{C} D_{t}^{q}$ and ${ }^{L} D_{t}^{1-q}$ are the generalized fractional derivative in Caputo and Riemann-Liouville senses, $B^{H}=\left\{B^{H}(t), t \in J\right\}$ is a cylindrical fractional Brownian motion with Hurst parameter $H \in\left(\frac{1}{2}, 1\right)$ defined on a filtered complete probability space $\left(\Omega, \mathcal{F},\left\{\mathcal{F}_{t}\right\}_{t \geq 0}, P\right)$. The operators $M: D(M) \subset X \longrightarrow X, L: D(L) \subset X \longrightarrow X$ and $V: D(V) \subset X \longrightarrow X, \Delta M$ is a bounded linear operator in $X$. The control function $u(\cdot)$ takes values in $L_{\mathcal{F}}^{2}(J, U)$, the Hilbert space of admissible control functions for a separable Hilbert space $U$ and $B$ is a bounded linear operator from $U$ to $X . f: J \times X \rightarrow X, g_{1}: J \rightarrow$ $L_{2}^{0}$ and $g_{2}: J \rightarrow L_{2}^{0}$ are appropriate Lipschitz type functions satisfying certain conditions to be specified later; $x_{0}$ is an $\mathcal{F}_{0}$-measurable random variable independent of $B_{1}^{H}$ and $B_{2}^{H}$.

The notion of controllability has played a central role throughout the history of modern control theory. Moreover, approximate controllable systems are more prevalent and fundamental concepts in deterministic and stochastic control theory. often approximate controllability is completely adequate in applications. Therefore, various approximate controllability problems for different kinds of nonlinear fractional dynamical systems in infinite dimensional spaces have been investigated in many publications; see [1,2] and references therein.

Stochastic differential equations are generalization of deterministic differential equations that incorporate a "noise term." These equations can be useful in many applications where we assume that there are deterministic changes combined with noisy fluctuations. Also, the study of stochastic differential equations has attracted great interest because of its applications in characterizing many problems in physics, biology,chemistry, mechanics, and so on. In finance and insurance, one has to deal with events such as corporate defaults, operational failures, or insured accidents, the theory and applications of stochastic differential equations in infinite-dimensional spaces have received much attention, (see [3-7] and the references therein).

On the other hand, some real world problems in science and engineering can be modeled by stochastic differential equations driven by fractional Brownian motion ( $\mathrm{fBm}$, for short). In particular, many types of stochastic differential equations driven by fBm in infinite dimension received much attention, for example, Maslowski and Nualart [8] studied nonlinear stochastic evolution equations in a Hilbert space driven by cylindrical fractional Brownian motion with Hurst parameter $H>\frac{1}{2}$ and nuclear covariance operator using techniques of fractional calculus with semigroup estimates. Boufoussi and Hajji [9] proved the existence and uniqueness of mild solutions of a neutral stochastic differential equations with nite delay, driven by a fractional Brownian motion in a Hilbert space and established some conditions ensuring the exponential decay to zero in mean square for the mild solution.

Approximate controllability for fractional stochastic systems are well investigated, we refer to $[10,11]$ and references therein.

Kerboua et al. [12] proved the approximate controllability of Sobolev type non-local fractional stochastic dynamic systems in Hilbert spaces by using fixed point technique, fractional calculus, stochastic analysis, and methods adopted directly from deterministic control 
problems. Kerboua et al. [13] introduced a new notion called fractional stochastic nonlocal condition for establishing approximate controllability of class of fractional stochastic nonlinear differential equations of Sobolev type in Hilbert spaces using Hölder's inequality, fixed point technique, fractional calculus, stochastic analysis and methods adopted directly from deterministic control problems.

Fěckan et al. [14] presented the controllability results corresponding to two admissible control sets for fractional functional evolution equations of Sobolev type in Banach spaces with the help of two new characteristic solution operators and their properties, such as boundedness and compactness, the results are obtained by using Schauder fixed point theorem.

It should be mentioned that there is no work yet reported on the approximate controllability of Sobolev type perturbed control systems of fractional order. Motivated by this facts, our main objective is to study the approximate controllability for a class of Sobolev type nonlinear stochastic differential equations of fractional order (1.1). The result is obtained under the assumption that the associated linear system is approximately controllable. In particular, the controllability question is transformed to a fixed point problem for an appropriate nonlinear operator in a function space. For that we need to construct a suitable set of sufficient conditions.

A brief outline of this paper is given. In Sect. 2, we present ssome basic notations and preliminaries on the stochastic integrals with respect to $\mathrm{fBm}$ in Hilbert space. In Sect. 3, the approximate controllability results of stochastic perturbed system of fractional order (1.1) is investigated by means of fractional calculus, semigroup theory and control theory. The last section deal with an illustrative example and a discussion for possible future work in this direction.

\section{Preliminaries}

Throughout of this paper, we assume that $H \in\left(\frac{1}{2}, 1\right)$ unless otherwise specified. In this section, we briefly introduce some useful results about $\mathrm{fBm}$ and the corresponding stochastic integral taking values in a Hilbert space.

\subsection{Fractional Brownian motion}

We begin by recalling the definition of a fractional Brownian motion. Let $\left(\Omega, \mathcal{F},\left\{\mathcal{F}_{t}\right\}_{t \geq 0}, P\right)$ be a filtered complete probability space. A real-valued Gauss process $\left\{\beta^{H}(t), t \geq 0\right\}$ defined on $\left(\Omega, \mathcal{F},\left\{\mathcal{F}_{t}\right\}_{t \geq 0}, P\right)$ is called a fBm with Hurst parameter $H \in(0,1)$ if $\mathbb{E}\left[\beta^{H}(t)\right]=0$ and the covariance function is given by $R_{H}(t, s)=\mathbb{E}\left[\beta^{H}(t) \beta^{H}(s)\right]=\frac{1}{2}\left(|t|^{2 H}+|s|^{2 H}-\right.$ $\left.|t-s|^{2 H}\right), t, s \in \mathbb{R}$ (see [15]).

Let $T>0$. It is known that $\mathrm{fBm}\left\{\beta^{H}(t), t \geq 0\right\}$ with $H>\frac{1}{2}$ admits the Wiener integral representation of the following form $\beta^{H}(t)=\int_{0}^{t} K_{H}(t, s) d w(s)$, where $w=\{w(t), t \geq 0\}$ is a standard Brownian motion and the kernel $K_{H}(t, s)$ is given by

$$
K_{H}(t, s)=c_{H} \int_{s}^{t}(u-s)^{H-\frac{3}{3}}\left(\frac{u}{s}\right)^{H-\frac{1}{2}} d u, s<t,
$$

where $c_{H}=\sqrt{\frac{H(2 H-1)}{\beta\left(2-2 H, H-\frac{1}{2}\right)}}$ and $\beta(\cdot, \cdot)$ denotes the Beta function.

For any deterministic function $\psi \in L^{2}([0, T])$, the Wiener integral of $\psi$ with respect to $\beta^{H}$ is defined by 


$$
\int_{0}^{T} \psi(s) d \beta^{H}(s)=\int_{0}^{T}\left(K_{H}^{*} \psi\right)(s) d w(s),
$$

where $\left(K_{H}^{*} \varphi\right)(s)=\int_{s}^{T} \varphi(r) \frac{\partial K_{H}}{\partial r}(r, s) d r$.

\subsection{Stochastic integral with respect to $\mathrm{fBm}$}

Let $\left(\Omega, \mathcal{F},\left\{\mathcal{F}_{t}\right\}_{t \geq 0}, P\right)$ be a filtered complete probability space and and $\mathcal{F}_{t}=\mathcal{F}_{t}^{B_{1}^{H}, B_{2}^{H}}$, where $\mathcal{F}_{t}^{B_{1}^{H}, B_{2}^{H}}$ is the sigma algebra generated by $\left\{\left(B_{1}^{H}(s), B_{2}^{H}(s)\right): 0 \leq s \leq t\right\}$.

Let $U=\left(U,\langle\cdot \cdot\rangle_{U},\|\cdot\|_{U}\right)$ and $Y=\left(Y,\langle\cdot \cdot\rangle_{Y},\|\cdot\|_{Y}\right)$ be real separable Hilbert spaces. Denote by $\mathcal{L}(U, Y)$ the space of bounded linear operators from $U$ to $Y$. A $Y$-valued, $\mathcal{F}_{t^{-}}$ adapted fBms can be defined by

$$
\begin{aligned}
& B_{1}^{H}(t)=\sum_{n=1}^{\infty} \sqrt{\lambda_{1, n}} e_{1, n} \beta_{1, n}^{H}(t), \\
& B_{2}^{H}(t)=\sum_{n=1}^{\infty} \sqrt{\lambda_{2, n}} e_{2, n} \beta_{2, n}^{H}(t),
\end{aligned}
$$

where $\left\{\beta_{1, n}^{H}, \beta_{2, n}^{H}\right\}_{n \geq 1}$ are sequences of independent fBms with the same Hurst parameter $H \in$ $\left(\frac{1}{2}, 1\right),\left\{e_{1, n} ; e_{2, n}\right\}_{n \geq 1}$ are complete orthonormal basis in $Y,\left\{\lambda_{1, n}, \lambda_{2, n}\right\}_{n \geq 1}$ are a bounded sequences of non-negative real numbers satisfying $Q e_{1, n}=\lambda_{1, n} e_{1, n}, Q e_{2, n}=\lambda_{2, n} e_{2, n}$ and $Q$ is non-negative self-adjoint trace class operator with $\operatorname{Tr} Q=\sum_{n=1}^{\infty} \lambda_{i, n}<+\infty$ for $i=1,2$.

Let $L_{2}^{0}(Y, X)$ denote the space of all $\psi \in \mathcal{L}(Y, X)$ such hat $\psi Q^{\frac{1}{2}}$ is a Hilbert-Schmidt operator. The norm is defined by $\|\psi\|_{L_{2}^{0}}^{2}=\sum_{n=1}^{\infty} \sqrt{\lambda_{n}} e_{n} \beta_{n}^{H}(t)$. Generally, $\psi$ is called a $Q$-Hilbert-Schmidt operator from $Y$ to $X$.

Let $\psi: J \rightarrow L_{2}^{0}(Y, X)$ such that

$$
\sum_{n=1}^{\infty}\left\|K_{H}^{*}\left(\psi Q^{\frac{1}{2}} e_{n}\right)\right\|_{L_{2}^{0}}^{2}<\infty .
$$

Then the stochastic integral of $\psi$ with respect to $\mathrm{fBm} B^{H}$ is defined by

$$
\begin{aligned}
\int_{0}^{t} \psi(s) d B^{H}(s) & =\sum_{n=1}^{\infty} \int_{0}^{t} \psi(s) Q^{\frac{1}{2}} e_{n} d \beta_{n}^{H}(s) \\
& =\sum_{n=1}^{\infty} \int_{0}^{t}\left(K_{H}^{*}\left(\psi Q^{\frac{1}{2}} e_{n}\right)\right)(s) d w(s) .
\end{aligned}
$$

Lemma 2.1 (see [9]) If $\psi: J \rightarrow L_{2}^{0}(Y, X)$ satisfying

$$
\sum_{n=1}^{\infty}\left\|\psi Q^{\frac{1}{2}} e_{n}\right\|_{L^{\frac{1}{H}}(J, X)}^{2}<\infty
$$

and for $u, v \in J$ with $v>u$, then

$$
\mathbb{E}\left\|\int_{u}^{v} \psi(s) d B^{H}(s)\right\|^{2} \leq c H(2 H-1)(v-u)^{2 H-1} \sum_{n=1}^{\infty} \int_{u}^{v}\left\|\psi(s) Q^{\frac{1}{2}} e_{n}\right\|^{2} d s,
$$


where $c=c(H)$. If, in addition, $\sum_{n=1}^{\infty}\left\|\psi(t) Q^{\frac{1}{2}} e_{n}\right\|$ is uniformly convergent for $t \in J$, then

$$
\mathbb{E}\left\|\int_{u}^{v} \psi(s) d B^{H}(s)\right\|^{2} \leq c H(2 H-1)(v-u)^{2 H-1} \int_{u}^{v}\|\psi(s)\|_{L_{2}^{0}}^{2} d s .
$$

Let $L^{2}\left(\mathcal{F}_{T}, X\right)$ be the Hilbert space of all $\mathcal{F}_{T}$-measurable square integrable random variables with values in the Hilbert space X. Let $L_{2}^{\mathcal{F}}(J, X)$ is the Hilbert space of all square integrable and $\mathcal{F}_{t}$-adapted processes with values in $X$. Let $\mathcal{C}_{T}$ denote the Banach space of all $X$-valued $\mathcal{F}_{t}$-adapted processes $\zeta(t, \omega): J \times \Omega \rightarrow X$, which are continuous in $t$ for a.e. fixed $\omega \in \Omega$ and satisfy

$$
\|\zeta\|_{\mathcal{C}_{T}}=E\left(\sup _{t \in J}\|\zeta(t, \omega)\|_{X}^{2}\right)^{\frac{1}{2}}
$$

Lemma 2.2 (see [16]) Let $M$ be the infinitesimal generator of an analytic semigroup $\{S(t), t \geq 0\}$ on a Hilbert space $X$. If $\Delta M$ is a bounded linear operator on $X$ then $(M+\Delta M)$ is the infinitesimal generator of an analytic semigroup $\{\tilde{S}(t), t \geq 0\}$ on $X$.

The operators $M+\Delta M: D(M+\Delta M) \subset X \rightarrow X, L: D(L) \subset X \rightarrow X$ and $V: D(V) \subset X \rightarrow X$ satisfy the following conditions:

(A1) $L,(M+\Delta M)$ and $V$ are closed linear operators.

(A2) $D(V) \subset D(L) \subset D(M+\Delta M)$ and $L$ and $V$ are bijective.

(A3) $L^{-1}: X \rightarrow D(L) \subset X$ and $V^{-1}: X \rightarrow D(V) \subset X$ are linear, bounded, and compact operators.

From (A3), we deduce that $L^{-1}$ is bounded operators. Note (A3) also implies that $L$ is closed since the fact: $L^{-1}$ is closed and injective, then its inverse is also closed. It comes from (A1)-(A3) and the closed graph theorem, we obtain the boundedness of the linear operator $(M+\Delta M) L^{-1}: X \rightarrow X$. Consequently, $(M+\Delta M) L^{-1}$ generates a semigroup $\left\{\tilde{S}(t)=e^{(M+\Delta M) L^{-1} t}, t \geq 0\right\}$. We suppose that $K_{0}=\sup _{t \geq 0}\|\tilde{S}(t)\|<\infty$, and for short, we denote by $C_{0}=\left\|L^{-1}\right\|$ and $C_{1}=\left\|V^{-1}\right\|$.

Now, we recall the following known definitions on the fractional integral and derivative.

Definition 2.1 The fractional integral of order $\alpha>0$ of a function $f \in L^{1}\left([a, b], \mathbb{R}^{+}\right)$is given by

$$
I_{a}^{\alpha} f(t)=\frac{1}{\Gamma(\alpha)} \int_{a}^{t}(t-s)^{\alpha-1} f(s) d s,
$$

where $\Gamma$ is the gamma function. If $a=0$, we can write $I^{\alpha} f(t)=\left(g_{\alpha} * f\right)(t)$, where

$$
g_{\alpha}(t)= \begin{cases}\frac{1}{\Gamma(\alpha)} t^{\alpha-1}, & t>0, \\ 0, & t \leq 0,\end{cases}
$$

and as usual, $*$ denotes the convolution of functions. Moreover, $\lim _{\alpha \rightarrow 0} g_{\alpha}(t)=\delta(t)$, with $\delta$ the delta Dirac function.

Definition 2.2 The Riemann-Liouville derivative of order $n-1<\alpha<n, n \in \mathbb{N}$, for a function $f \in C([0,+\infty))$ is given by

$$
{ }^{L} D^{\alpha} f(t)=\frac{1}{\Gamma(n-\alpha)} \frac{d^{n}}{d t^{n}} \int_{0}^{t} \frac{f(s)}{(t-s)^{\alpha+1-n}} d s, \quad t>0 .
$$


Definition 2.3 The Caputo derivative of order $n-1<\alpha<n, n \in \mathbb{N}$, for a function $f \in C([0,+\infty))$ is given by

$$
{ }^{C} D^{\alpha} f(t)={ }^{L} D^{\alpha}\left(f(t)-\sum_{k=0}^{n-1} \frac{t^{k}}{k !} f^{(k)}(0)\right), \quad t>0 .
$$

Remark 2.1 The following properties hold. Let $n-1<\alpha<n, \quad n \in \mathbb{N}$

(i) If $f(t) \in C^{n}([0, \infty))$, then

$$
{ }^{C} D^{\alpha} f(t)=\frac{1}{\Gamma(n-\alpha)} \int_{0}^{t} \frac{f^{(n)}(s)}{(t-s)^{\alpha+1-n}} d s=I^{n-\alpha} f^{(n)}(s), \quad t>0 .
$$

(ii) The Caputo derivative of a constant is equal to zero.

(iii) The Riemann-Liouville derivative of a constant function is given by

$$
{ }^{L} D_{a^{+}}^{\alpha} C=\frac{C}{\Gamma(1-\alpha)}(x-a)^{-\alpha} .
$$

If $f$ is an abstract function with values in $X$, then integrals which appear in Definitions 2.1-2.3 are taken in Bochner's sense.

According to previous definitions, it is suitable to rewrite problem (1.1)-(1.2) as the equivalent integral equation

$$
\begin{aligned}
L x(t)= & L x(0)+\frac{1}{\Gamma(q)} \int_{0}^{t}(t-s)^{q-1}[(M+\Delta M) x(s)+B u(s)+f(s, x(s))] d s \\
& +\frac{1}{\Gamma(q)} \int_{0}^{t}(t-s)^{q-1} g_{1}(s) d B_{1}^{H}(s)
\end{aligned}
$$

Remark 2.2 We note that:

(a) For the nonlocal condition, the function $x(0)$ is dependent on $t$.

(b) ${ }^{L} D_{t}^{1-q}[V x(0)]$ is well defined, i.e., if $q=1$ and $V$ is the identity, then (1.2) reduces to the usual nonlocal condition..

(c) The function $x(0)$ takes the form

$$
V^{-1} x_{0}+\frac{1}{\Gamma(1-q)} \int_{0}^{t}(t-s)^{-q} V^{-1} g_{2}(s) d B_{2}^{H}(s),
$$

where $\left.V x(0)\right|_{t=0}=x_{0}$.

(d) The explicit and implicit integrals given in (2.1) exist (taken in Bochner's sense).

Definition 2.4 A stochastic process $x \in H_{2}(J, X)$ is a mild solution of (1.1)-(1.2) if for each control $u \in L_{\Gamma}^{2}(J, U)$, it satisfies,

1. $x(0) \in L^{2}(\Gamma, X)$, where $x(0)=V^{-1} x_{0}+\frac{1}{\Gamma(1-q)} \int_{0}^{t}(t-s)^{-q} V^{-1} g_{2}(s) d B_{2}^{H}(s)$ and $\left.V x(0)\right|_{t=0}=x_{0}$;

2. $x(t) \in X$ has cádlág paths on $t \in J$ almost surely and for each $t \in J, x(t)$ satisfies the integral equation 


$$
\begin{aligned}
x(t)= & \tilde{\mathcal{S}}_{q}(t) L V^{-1}\left[x_{0}+\frac{1}{\Gamma(1-q)} \int_{0}^{t}(t-s)^{-q} g_{2}(s) d B_{2}^{H}(s)\right] \\
& +\int_{0}^{t}(t-s)^{q-1} \tilde{\mathcal{T}}_{q}(t-s)[B u(s)+f(s, x(s))] d s \\
& +\int_{0}^{t}(t-s)^{q-1} \tilde{\mathcal{T}}_{q}(t-s) g_{1}(s) d B_{1}^{H}(s),
\end{aligned}
$$

where $\tilde{\mathcal{S}}_{q}(t) x=\int_{0}^{+\infty} L^{-1} h_{q}(s) \tilde{S}\left(t^{q} s\right) x d s$ and $\tilde{\mathcal{T}}_{q}(t) x=q \int_{0}^{+\infty} L^{-1} s h_{q}(s) \tilde{S}\left(t^{q} s\right) x d s$.

Here, $\tilde{S}(t)$ is a $C_{0}$-semigroup generated by a linear operator $(M+\Delta M) L^{-1}: X \rightarrow X$, $h_{q}$ is a probability density function defined on $(0, \infty)$, that is $h_{q}(s) \geq 0, s \in(0, \infty)$ and $\int_{0}^{\infty} h_{q}(s) d s=1$.

The following lemma follows from the results in [16-20] and will be used throughout this paper.

Lemma 2.3 (see [21]) The operators $\tilde{\mathcal{S}}_{q}(t)$ and $\tilde{\mathcal{T}}_{q}(t)$ have the following properties:

1. For any fixed $t \geq 0, \tilde{\mathcal{S}}_{q}(t)$ and $\tilde{\mathcal{T}}_{q}(t)$ are linear and bounded operators in $X$,

$$
\text { i.e.for any } x \in X_{\alpha}, \quad\left\|\tilde{\mathcal{S}}_{q}(t) x\right\| \leq C_{0} K_{0}\|x\|, \quad\left\|\tilde{\mathcal{T}}_{q}(t) x\right\| \leq \frac{C_{0} K_{0}}{\Gamma(q)}\|x\| .
$$

2. The operators $\left\{\tilde{\mathcal{S}}_{q}(t): t \geq 0\right\}$ and $\left\{\tilde{\mathcal{T}}_{q}(t): t \geq 0\right\}$ are strongly continuous.

We impose the following assumptions on the data of the problem (1.1)-(1.2).

(H1) The functions $f: J \times X \rightarrow X$ satisfy linear growth and Lipschitz conditions. Moreover, there exist positive constants $N_{1}, N_{2}>0$ such that

$$
\|f(t, x)-f(t, y)\|^{2} \leq N_{1}\|x-y\|^{2},\|f(t, x)\|^{2} \leq N_{2}\left(1+\|x\|^{2}\right),
$$

(H2) The function $g_{i}: J \rightarrow L_{2}^{0}$ for $i=1$, 2, satisfies $\int_{0}^{T}\left\|g_{i}(s)\right\|_{L_{2}^{0}}^{2} d s<\infty$.

(H3) The linear stochastic system is approximately controllable on J.

For each $0 \leq t<T$, the operator $z\left(z I+\Psi_{0}^{T}\right)^{-1} \rightarrow 0$ in the strong operator topology as $z \rightarrow 0^{+}$, where $\Psi_{0}^{T}=\int_{0}^{T}(T-s)^{2(q-1)} \tilde{\mathcal{T}}_{q}(T-s) B B^{*} \tilde{\mathcal{T}}_{q}^{*}(T-s) d s$ is the controllability Gramian, here $B^{*}$ denotes the adjoint of $B$ and $\tilde{\mathcal{T}}_{q}^{*}(t)$ is the adjoint of $\tilde{\mathcal{T}}_{q}(t)$.

Observe that Sobolev type linear fractional deterministic control system

$$
\begin{aligned}
& { }^{C} D_{t}^{q}[L x(t)]=(M+\Delta M) x(t)+B u(t), t \in J, \\
& x(0)=x_{0},
\end{aligned}
$$

corresponding to (1.1)-(1.2) is approximately controllable on $J$ iff the operator $z(z I+$ $\left.\Psi_{0}^{T}\right)^{-1} \rightarrow 0$ strongly as $z \rightarrow 0^{+}$. The approximate controllability for linear fractional deterministic control system (2.3)-(2.4) is a natural generalization of approximate controllability of linear first order control system ( $q=1$ and $L$ is the identity) [22].

Definition 2.5 System (1.1)-(1.2) is approximately controllable on $J$ if $\overline{\Re(T)}=L^{2}\left(\Omega, \Gamma_{T}\right.$, $X)$, where

$$
\Re(T)=\left\{x(T)=x(T, u): u \in L_{\Gamma}^{2}(J, U)\right\},
$$

here $L_{\Gamma}^{2}(J, U)$, is the closed subspace of $L_{\Gamma}^{2}(J \times \Omega ; U)$, consisting of all $\Gamma_{t}$ adapted, $U$ -valued stochastic processes.

The following lemma is required to define the control function [23]. 
Lemma 2.4 For any $\tilde{x}_{T} \in L^{2}\left(\Gamma_{T}, X\right)$, there exists $\tilde{\varphi} \in L_{\Gamma}^{2}\left(\Omega ; L^{2}\left([0, T] ; L_{2}^{0}\right)\right)$ such that $\tilde{x}_{T}=E \tilde{x}_{T}+\int_{0}^{T} \tilde{\varphi}(s) d B^{H}(s)$. Now for any $\lambda>0$ and $\tilde{x}_{T} \in L^{2}\left(\Gamma_{T}, X\right)$, we define the control function in the following form

$$
\begin{aligned}
& u^{\lambda}(t, x)=B^{*}(T-t)^{q-1} \tilde{\mathcal{T}}_{q}^{*}(T-t)\left[\left(z I+\Psi_{0}^{T}\right)^{-1}\right. \\
& \quad\left\{E \tilde{x}_{T}-\tilde{\mathcal{S}}_{q}(T) L V^{-1}\left(x_{0}+\frac{1}{\Gamma(1-q)} \int_{0}^{t}(t-s)^{-q} g_{2}(s) d B_{2}^{H}(s)\right)\right\} \\
& \left.+\int_{0}^{t}\left(z I+\Psi_{0}^{T}\right)^{-1} \tilde{\varphi}(s) d B_{1}^{H}(s)\right] \\
& -B^{*}(T-t)^{q-1} \tilde{\mathcal{T}}_{q}^{*}(T-t) \int_{0}^{t}\left(z I+\Psi_{0}^{T}\right)^{-1}(T-s)^{q-1} \tilde{\mathcal{T}}_{q}(T-s) f(s, x(s)) d s \\
& -B^{*}(T-t)^{q-1} \tilde{\mathcal{T}}_{q}^{*}(T-t) \int_{0}^{t}\left(z I+\Psi_{0}^{T}\right)^{-1}(T-s)^{q-1} \tilde{\mathcal{T}}_{q}(T-s) g_{1}(s) d B_{1}^{H}(s) .
\end{aligned}
$$

Lemma 2.5 There exist positive real constants $\hat{M}, \hat{N}$ such that, for all $x, y \in H_{2}$, we have

$$
\begin{aligned}
E\left\|u^{\lambda}(t, x)-u^{\lambda}(t, y)\right\|^{2} & \leq \hat{M} E\|x(t)-y(t)\|^{2}, \\
E\left\|u^{\lambda}(t, x)\right\|^{2} & \leq \hat{N}\left(\frac{1}{T}+E\|x(t)\|^{2}\right) .
\end{aligned}
$$

Proof The proof of this lemma similar to the proof of the Lemma 2.4 (see [13]).

\section{Approximate controllability}

In this section, we formulate and prove conditions for the existence and approximate controllability results of the nonlocal fractional stochastic perturbed control system of Sobolev type (1.1)-(1.2) using the contraction mapping principle.

Theorem 3.1 Assume assumptions (H1)-(H3) are satisfied. Then, for all $T>0$, the system (1.1)-(1.2) has a mild solution on $[0, T]$.

Proof For any $\lambda>0$, define the operator $\mathrm{F}_{\lambda}: \mathrm{H}_{2} \rightarrow \mathrm{H}_{2}$ by

$$
\begin{aligned}
F_{\lambda} x(t)= & \tilde{\mathcal{S}}_{q}(t) L V^{-1}\left[x_{0}+\frac{1}{\Gamma(1-q)} \int_{0}^{t}(t-s)^{-q} g_{2}(s) d B_{2}^{H}(s)\right] \\
& +\int_{0}^{t}(t-s)^{q-1} \tilde{\mathcal{T}}_{q}(t-s)\left[B u^{\lambda}(s, x)+f(s, x(s))\right] d s \\
& +\int_{0}^{t}(t-s)^{q-1} \tilde{\mathcal{T}}_{q}(t-s) g_{1}(s) d B_{1}^{H}(s) .
\end{aligned}
$$

It will be shown that, for all $\lambda>0$, the operator $F_{\lambda}$ has a fixed point. This fixed point is then a solution of Eqs. (1.1)-(1.2). To prove this result, we divide the subsequent proof into two steps.

Step 1 For arbitrary $x \in H_{2}$, let us prove that $t \rightarrow F_{\lambda}(x)(t)$ is continuous on the interval $J$ in $L^{2}$-sense.

Let $0<t<t+h<T$, where $t, t+h \in[0, T]$, and let $|h|$ be sufficiently small. 
Then for any fixed $x \in H_{2}$, it follows from Holder's inequality and the assumptions on the theorem that

$$
\begin{aligned}
& \mathbb{E}\left\|F_{\lambda}(x)(t+h)-F_{\lambda}(x)(t)\right\|^{2} \\
& \leq 4 \mathbb{E}\left\|\left(\tilde{\mathcal{S}}_{q}(t+h)-\tilde{\mathcal{S}}_{q}(t)\right) L V^{-1}\left[\frac{1}{\Gamma(1-q)} \int_{t}^{t+h}(t-s)^{-q} g_{2}(s) d B_{2}^{H}(s)\right]\right\|^{2} \\
& +4 \mathbb{E}\left\|\int_{0}^{t}(t-s)^{q-1}\left(\tilde{\mathcal{T}}_{q}(t+h-s)-\tilde{\mathcal{T}}_{q}(t-s)\right) B u^{\lambda}(s, x) d s\right\|^{2} \\
& +4 \mathbb{E}\left\|\int_{t}^{t+h}(t-s)^{q-1} \tilde{\mathcal{T}}_{q}(t+h-s) B u^{\lambda}(s, x) d s\right\|^{2} \\
& +4 \mathbb{E}\left\|\int_{0}^{t}(t-s)^{q-1}\left(\tilde{\mathcal{T}}_{q}(t+h-s)-\tilde{\mathcal{T}}_{q}(t-s)\right) f(s, x(s)) d s\right\|^{2} \\
& +4 \mathbb{E}\left\|\int_{t}^{t+h}(t-s)^{q-1} \tilde{\mathcal{T}}_{q}(t+h-s) f(s, x(s)) d s\right\|^{2} \\
& +4 \mathbb{E}\left\|\int_{0}^{t}(t-s)^{q-1}\left(\tilde{\mathcal{T}}_{q}(t+h-s)-\tilde{\mathcal{T}}_{q}(t-s)\right) g_{1}(s) d B_{1}^{H}\right\|^{2} \\
& +4 \mathbb{E}\left\|\int_{t}^{t+h}(t-s)^{q-1} \tilde{\mathcal{T}}_{q}(t+h-s) g_{1}(s) d B_{1}^{H}\right\|^{2} \\
& \leq 4 C_{1}\|L\|^{2} E\left\|\left(\tilde{\mathcal{S}}_{q}(t+h)-\tilde{\mathcal{S}}_{q}(t)\right)\left[\frac{1}{\Gamma(1-q)} \int_{t}^{t+h}(t-s)^{-q} g_{2}(s) d B_{2}^{H}(s)\right]\right\|^{2} \\
& +4 \frac{t^{2 q-1}}{2 q-1} \int_{0}^{t} E\left\|\left(\tilde{\mathcal{T}}_{q}(t+h-s)-\tilde{\mathcal{T}}_{q}(t-s)\right) B u^{\lambda}(s, x)\right\|^{2} d s \\
& +4 \frac{h^{2 q-1}}{1-2 q}\left(\frac{C_{0} K_{0}}{\Gamma(q)}\right)^{2}\|B\|^{2} \int_{t}^{t+h} E\left\|u^{\lambda}(s, x)\right\|^{2} d s \\
& +4 \frac{t^{2 q-1}}{2 q-1} \int_{0}^{t} E\left\|\left(\tilde{\mathcal{T}}_{q}(t+h-s)-\tilde{\mathcal{T}}_{q}(t-s)\right) f(s, x(s))\right\|^{2} d s \\
& +4 \frac{h^{2 q-1}}{1-2 q}\left(\frac{C_{0} K_{0}}{\Gamma(q)}\right)^{2} \int_{t}^{t+h} E\|f(s, x(s))\|^{2} d s \\
& +8 \frac{t^{2 q-1}}{2 q-1} H t^{2 H-1} \int_{0}^{t} E\left\|\left(\tilde{\mathcal{T}}_{q}(t+h-s)-\tilde{\mathcal{T}}_{q}(t-s)\right) g_{1}(s)\right\|_{L_{2}^{0}}^{2} d s \\
& +8 \frac{h^{2 q-1}}{1-2 q} H h^{2 H-1}\left(\frac{C_{0} K_{0}}{\Gamma(q)}\right)^{2} \int_{t}^{t+h} E\left\|g_{1}(s)\right\|_{L_{2}^{0}}^{2} d s .
\end{aligned}
$$

Hence using the strong continuity of the operators $\left\{\tilde{\mathcal{S}}_{q}(t), \tilde{\mathcal{T}}_{q}(t)\right\}_{t \geq 0}$ and Lebesgue's dominated convergence theorem, we conclude that the right-hand side of the above inequalities tends to zero as $h \longrightarrow 0$.

Thus, we conclude $F_{\lambda}(x)(t)$ is continuous from the right of [0,T). A similar argument shows that it is also continuous from the left of $(0, T]$.

Step 2 Now, we are going to show that $F_{\lambda}$ is a contraction mapping in $H_{2}$. 
Let $x, y \in H_{2}$, we obtain for any fixed $t \in[0, T]$

$$
\begin{aligned}
& \mathbb{E}\left\|F_{\lambda}(x)(t)-F_{\lambda}(y)(t)\right\|^{2} \leq 2 \mathbb{E}\left\|\int_{0}^{t}(t-s)^{q-1} \mathcal{T}_{q}(t-s) B\left[u^{\lambda}(s, x)-u^{\lambda}(s, y)\right] d s\right\|^{2} \\
& \quad+2 \mathbb{E}\left\|\int_{0}^{t}(t-s)^{q-1} \mathcal{T}_{q}(t-s)[f(s, x(s))-f(s, y(s))] d s\right\|^{2}
\end{aligned}
$$

Using assumptions (H1)-(H2), Lemma 2.5, and standard computations yield

$$
\begin{aligned}
& \mathbb{E}\left\|F_{\lambda}(x)(t)-F_{\lambda}(y)(t)\right\|^{2} \leq 2 \frac{T^{2 q-1}}{2 q-1}\left(\frac{C_{0} K_{0}}{\Gamma(q)}\right)^{2}\|B\|^{2} \hat{M} \int_{0}^{t} \mathbb{E}\|(x(t)-y(t))\|^{2} d s \\
& +2 \frac{T^{2 q-1}}{2 q-1}\left(\frac{C_{0} K_{0}}{\Gamma(q)}\right)^{2} N_{1}^{2} E\left\|\int_{0}^{t} \mathbb{E}\right\|(x(t)-y(t)) \|^{2} d s
\end{aligned}
$$

Hence, we obtain a positive real constant $\gamma(\lambda)$ such that

$$
\sup _{t \in J} \mathbb{E}\left\|F_{\lambda}(x)(t)-F_{\lambda}(y)(t)\right\|^{2} \leq \gamma(\lambda) \sup _{t \in J} \mathbb{E}\|x(t)-y(t)\|^{2}
$$

for all $t \in J$ and all $x, y \in H_{2}$. For any natural number $n$, it follows from successive iteration of above inequality (3.2) that, by taking the supremum over $J$,

$$
\left\|F_{\lambda}^{n}(x)(t)-F_{\lambda}^{n}(y)(t)\right\|_{H_{2}}^{2} \leq \frac{\gamma^{n}(\lambda)}{n !}\|x-y\|_{H_{2}}^{2}
$$

For any fixed $\lambda>0$, for sufficiently large $n, \frac{\gamma^{n}(\lambda)}{n !}<1$. It follows from (3.3) that $F_{\lambda}^{n}$ is a contraction mapping, so that the contraction principle ensures that the operator $F_{\lambda}$ has a unique fixed point $x_{\lambda}$ in $H_{2}$, which is a mild solution of (1.1)-(1.2).

Theorem 3.2 Assume that the assumptions (H1)-(H3) hold. Further, if the functions $f, g_{1}$ and $g_{2}$ are uniformly bounded and $\left\{\tilde{\mathcal{T}}_{q}: t \geq 0\right\}$ is compact, then the system (1.1)-(1.2) is approximately controllable on $\mathrm{J}$.

Proof Let $x_{\lambda}$ be a fixed point of $F_{\lambda}$. By using the stochastic Fubini theorem, it can be easily seen that

$$
\begin{aligned}
x_{\lambda}(T)= & \tilde{x}_{T}-z\left(z I+\Psi_{0}^{T}\right)^{-1}\left\{E \tilde{x}_{T}-\tilde{\mathcal{S}}_{q}(T) L V^{-1}\left[x_{0}+\frac{1}{\Gamma(1-q)} \int_{0}^{t}(t-s)^{-q} g_{2}(s) d B_{2}^{H}(s)\right]\right\} \\
& +z \int_{0}^{T}\left(z I+\Psi_{0}^{T}\right)^{-1}(T-s)^{q-1} \tilde{\mathcal{T}}_{q}(T-s) f\left(s, x_{\lambda}(s)\right) d s \\
& +z \int_{0}^{T}\left(z I+\Psi_{0}^{T}\right)^{-1}\left[(T-s)^{q-1} \tilde{\mathcal{T}}_{q}(T-s) g_{1}(s)-\tilde{\varphi}(s)\right] d B_{1}^{H}(s) .
\end{aligned}
$$

It follows from the assumption on $f$ that there exists $\hat{D}>0$ such that

$$
\left\|f\left(s, x_{\lambda}(s)\right)\right\|^{2} \leq \hat{D}
$$

for all $(s, \omega) \in J \times \Omega$. Then there is a subsequence still denoted by $f\left(s, x_{\lambda}(s)\right)$ which converges weakly to, say, to $f(s)$ in $X \times L_{2}^{0}$.

From the above equation, we have

$$
\begin{aligned}
& \mathbb{E}\left\|x_{\lambda}(T)-\tilde{x}_{T}\right\|^{2} \\
& \quad \leq 6 \mathbb{E}\left(\left\|z\left(z I+\Psi_{0}^{T}\right)^{-1}\left(E \tilde{x}_{T}-\tilde{\mathcal{S}}_{q}(T) L V^{-1} x_{0}\right)\right\|^{2}\right)
\end{aligned}
$$




$$
\begin{aligned}
& +12 H t^{2 H-1}\left(\int_{0}^{T}(T-s)^{-q}\left\|z\left(z I+\Psi_{0}^{T}\right)^{-1} \tilde{\mathcal{S}}_{q}(T) L V^{-1} \frac{1}{\Gamma(1-q)}\right\|^{2}\left\|g_{2}(s)\right\|_{L_{2}^{0}}^{2} d s\right) \\
& +6 \mathbb{E}\left(\int_{0}^{T}(T-s)^{q-1}\left\|z\left(z I+\Psi_{0}^{T}\right)^{-1} \widetilde{\varphi}(s)\right\|_{L_{2}^{0}}^{2} d s\right) \\
& +6 \mathbb{E}\left(\int_{0}^{T}(T-s)^{q-1}\left\|z\left(z I+\Psi_{0}^{T}\right)^{-1}\right\|\left\|\tilde{\mathcal{T}}_{q}(T-s)\left(f\left(s, x_{\lambda}(s)\right)-f(s)\right)\right\| d s\right)^{2} \\
& +6 \mathbb{E}\left(\int_{0}^{T}(T-s)^{q-1}\left\|z\left(z I+\Psi_{0}^{T}\right)^{-1} \tilde{\mathcal{T}}_{q}(T-s) f(s)\right\| d s\right)^{2} \\
& +12 H t^{2 H-1}\left(\int_{0}^{T}(T-s)^{q-1}\left\|z\left(z I+\Psi_{0}^{T}\right)^{-1} \tilde{\mathcal{T}}_{q}(T-s) g_{1}(s)\right\|_{L_{2}^{0}}^{2} d s\right) .
\end{aligned}
$$

On the other hand, by assumption (H3), for all $0 \leq s<T$ the operator $z\left(z I+\Psi_{0}^{T}\right)^{-1} \rightarrow 0$ strongly as $z \rightarrow 0^{+}$and moreover $\left\|z\left(z I+\Psi_{0}^{T}\right)^{-1}\right\| \leq 1$. Thus, by the Lebesgue dominated convergence theorem and the compactness of both $\tilde{\mathcal{S}}_{q}$ and $\tilde{\mathcal{T}}_{q}$ implies that $E\left\|x_{\lambda}(T)-\tilde{x}_{\lambda}\right\|^{2} \rightarrow$ 0 as $z \rightarrow 0^{+}$. Hence, we conclude the approximate controllability of (1.1)-(1.2).

\section{Example}

In this section, we present an example to illustrate our main result.

Let us consider the following Sobolev type fractional stochastic partial differential equation with control driven by a fractional Brownian motion:

$$
\begin{gathered}
\left.{ }^{C} D_{t}^{2 / 3}\left[x(t, z)-x_{z z}(t, z)\right)\right]-\frac{\partial^{2}}{\partial z^{2}} x(t, z)=\mu(t, z)+\tilde{f}(t, x(t, z)) \\
\quad+\tilde{g}_{1}(t, z) \frac{d \tilde{B}_{1}^{H}(t)}{d t} \\
x(0, z)=\frac{\partial^{2}}{\partial z^{2}}\left[x_{0}(z)+\frac{1}{\Gamma(1 / 3)} \sum_{k=1}^{m} c_{k} \int_{0}^{t}(t-s)^{-2 / 3} g_{2}\left(t_{k}, z\right) \tilde{B}_{2}^{H}(s)\right], z \in[0,1] \\
x(0, t)=x(1, t)=0, t \in J
\end{gathered}
$$

where $0<q \leq 1,0<t_{1}<\cdots<t_{m}<T$ and $c_{k}$ are positive constants, $k=1, \ldots, m$; the functions $x(t)(z)=x(t, z), f(t, x(t))(z)=\tilde{f}(t, x(t, z)), g_{1}(t)(z)=\tilde{g}_{1}(t, z)$ and $g_{2}(t)(z)=\sum_{k=1}^{m} c_{k} g_{2}\left(t_{k}, z\right)$. The bounded linear operator $B: U \rightarrow X$ is defined by $B u(t)(z)=\mu(t, z), 0 \leq z \leq 1, u \in U ; \tilde{B}_{1}^{H}(t)$ and $\tilde{B}_{2}^{H}(t)$ are two sided one dimensional fractional Brownian motions with Hurst parameter $H \in(1 / 2,1)$.

Let $X=E=U=L^{2}[0,1]$, define the operators $L: D(L) \subset X \rightarrow X, M+\Delta M$ : $D(M+\Delta M) \subset X \rightarrow X$ and $V: D(V) \subset X \rightarrow X$ by $L x=x-x_{z z},(M+\Delta M) x=-x_{z z}$ and $V^{-1} x=x_{z z}$ where domains $D(L), D(M+\Delta M)$ and $D(V)$ are given by

$\left\{x \in X: x, x_{z}\right.$ are absolutely continuous, $\left.x_{z z} \in X, x(0)=x(1)=0\right\}$.

Then $L,(M+\Delta M)$ and $V$ can be written respectively as

$$
L x=\sum_{n=1}^{\infty}\left(1+n^{2}\right)\left(x, x_{n}\right) x_{n}, x \in D(L),
$$




$$
\begin{aligned}
(M+\Delta M) x & =\sum_{n=1}^{\infty}-n^{2}\left(x, x_{n}\right) x_{n}, x \in D(M+\Delta M), \\
V^{-1} x & =\sum_{n=1}^{\infty} n^{2}\left(x, x_{n}\right) x_{n}, x \in D(V),
\end{aligned}
$$

where $x_{n}(z)=(\sqrt{2 / \pi}) \sin n z, n=1,2, \ldots$ is the orthogonal set of eigen functions of $(M+\Delta M)$. Further, for any $x \in X$ we have

$$
\begin{aligned}
L^{-1} x & =\sum_{n=1}^{\infty} \frac{1}{1+n^{2}}\left(x, x_{n}\right) x_{n}, \\
(M+\Delta M) L^{-1} x & =\sum_{n=1}^{\infty} \frac{-n^{2}}{1+n^{2}}\left(x, x_{n}\right) x_{n},
\end{aligned}
$$

and

$$
\begin{aligned}
\tilde{S}(t) x & =\sum_{n=1}^{\infty} \exp \left(\frac{-n^{2} t}{1+n^{2}}\right)\left(x, x_{n}\right) x_{n} . \\
\tilde{\mathcal{T}}_{2 / 3}(t) x & =\frac{2}{3} \sum_{n=1}^{\infty} \frac{1}{1+n^{2}} \int_{0}^{+\infty} s h_{2 / 3}(s) \exp \left(\frac{-n^{2}}{1+n^{2}} t^{2 / 3} s\right) d s\left(x, x_{n}\right) x_{n} .
\end{aligned}
$$

It is easy to see that $L^{-1}$ is compact, bounded with $\left\|L^{-1}\right\| \leq 1$ and $(M+\Delta M) L^{-1}$ generates the above strongly continuous semigroup $\tilde{S}(t)$ on $X$ with $\|\tilde{S}(t)\| \leq e^{-t} \leq 1$. Therefore, with the above choices, the system (4.1)-(4.3) can be written as an abstract formulation of (2.1)(2.2) and thus Theorem 3.1 can be applied to guarantee the existence of mild solution of (4.1)-(4.3). Moreover, it can be easily seen that Sobolev type deterministic linear fractional control system corresponding to (4.1)-(4.3) is approximately controllable on $J$, which means that all conditions of Theorem 3.2 are satisfied. Thus, Sobolev type fractional stochastic partial differential equation with control driven by a fractional Brownian motion (4.1)-(4.3) is approximately controllable on $J$.

\section{Conclusion}

Sufficient conditions for the approximate controllability of a class of control systems described by Sobolev type nonlocal nonlinear fractional stochastic perturbed equations with fractional Brownian motion in Hilbert spaces are considered. Using fixed point technique, fractional calculations, stochastic integrals for fractional Brownian motion, and methods adopted directly from deterministic control problems. In particular, conditions are formulated and proved under the assumption that the approximate controllability of the fractional stochastic control nonlinear perturbed system is implied by the approximate controllability of its corresponding linear part. More precisely, the controllability problem is transformed into a fixed point problem for an appropriate nonlinear operator in a function space. The main used tools are the above required conditions, we guarantee the existence of a fixed point of this operator and study controllability of the considered systems.

Our future work will be focused on investigating the approximate controllability for fractional stochastic dynamical systems of Sobolev type with Lévy process and impulsive effects. Upon making some appropriate assumptions, by employing the ideas and techniques as in 
this paper, one can establish the approximate controllability results for a class of Sobolev type nonlocal nonlinear fractional stochastic dynamical systems with Lé vy process and impulsive effects in Hilbert spaces.

Acknowledgements The authors would like to express their sincere gratitude to the anonymous reviewers for their careful reading of the manuscript, as well as their comments that lead to a considerable improvement of the original manuscript.

\section{Compliance with ethical standards}

Conflict of interest The authors declare that there is no conflict of interests regarding the publication of this paper.

Open Access This article is distributed under the terms of the Creative Commons Attribution 4.0 International License (http://creativecommons.org/licenses/by/4.0/), which permits unrestricted use, distribution, and reproduction in any medium, provided you give appropriate credit to the original author(s) and the source, provide a link to the Creative Commons license, and indicate if changes were made.

\section{References}

1. Sakthivel, R., Ganesh, R., Suganya, R.: Approximate controllability of fractional neutral stochastic system with infinite delay. Rep. Math. Phys. 70, 291-311 (2012)

2. Sakthivel, R., Ganesh, R., Ren, Y., Anthoni, S.M.: Approximate controllability of nonlinear fractional dynamical systems. Commun. Nonlinear Sci. Numer. Simul. 18, 3498-3508 (2013)

3. Bashirov, A.E., Mahmudov, N.I.: On concepts of controllability for linear deterministic and stochastic systems. SIAM J. Control Optim. 37, 1808-1821 (1999)

4. Cao, J., Yang, Q., Huang, Z.: On almost periodic mild solutions for stochastic functional differential equations. Nonlinear Anal. Real World Appl. 13, 275-286 (2012)

5. Chang, Y.K., Zhao, Z.H., N'Guérékata, G.M., Ma, R.: Stepanov-like almost automorphy for stochastic processes and applications to stochastic differential equations. Nonlinear Anal. Real World Appl. 12, $130-1139$ (2011)

6. Mao, X.: Stochastic Differential Equations and Their Applications. Horwood, Chichester (1997)

7. Sakthivel, R., Revathi, P., Renc, Y.: Existence of solutions for nonlinear fractional stochastic differential equations. Nonlinear Anal. 81, 70-86 (2013)

8. Maslowski, B., Nualart, D.: Evolution equations driven by a fractional Brownian motion. J. Funct. Anal. 202, 277-305 (2003)

9. Boufoussi, B., Hajji, S.: Neutral stochastic functional differential equations driven by a fractional Brownian motion in a Hilbert space. Stat. Probab. Lett. 82, 1549-1558 (2012)

10. Mahmudov, N.I.: Approximate controllability of semilinear deterministic and stochastic evolution equations in abstract spaces. SIAM J. Control Optim. 42, 1604-1622 (2003)

11. Li, F., Liang, J., Xu, H.K.: Existence of mild solutions for fractional integrodifferential equations of Sobolev type with nonlocal conditions. J. Math. Anal. Appl. 391, 510-525 (2012)

12. Kerboua, M., Debbouche, A., Baleanu, D.: Approximate controllability of Sobolev type nonlocal fractional stochastic dynamic systems in Hilbert spaces. Abstr. Appl. Anal. Art. ID 262191 (2013)

13. Kerboua, M., Debbouche, A., Baleanu, D.: Approximate controllability of Sobolev type fractional stochastic nonlocal nonlinear differential equations in Hilbert spaces. Electron. J. Qual. Theory Differ. Equ. 58, $1-16(2014)$

14. Fěckan, M., Wang, J.R., Zhou, Y.: Controllability of fractional functional evolution equations of Sobolev type via characteristic solution operators. J. Optim. Theory. Appl. 156, 79-95 (2013)

15. Mandelbrot, B.B., Van Ness, J.W.: Fractional Brownian motions, fractional noises and applications. SIAM Rev. 10, 422-437 (1968)

16. El-Borai, M.M.: Some probability densities and fundamental solutions of fractional evolution equations. Chaos Solitons Fractals 14(3), 433-440 (2002)

17. El-Borai, M.M.: On some stochastic fractional integro-differential equations. Adv/. Dyn. Syst. Appl. 1(1), 49-57 (2006)

18. Liu, H., Chang, J.C.: Existence for a class of partial differential equations with nonlocal conditions. Nonlinear Anal. Theory Methods Appl. 70(9), 3076-3083 (2009) 
19. Wang, J., Zhou, Y.: A class of fractional evolution equations and optimal controls. Nonlinear Anal. Real World Appl. 12(1), 262-272 (2011)

20. Wang, R.N., Xiao, T.J., Liang, J.: A note on the fractional Cauchy problems with nonlocal initial conditions. Appl. Math. Lett. 24(8), 1435-1442 (2011)

21. Pazy, A.: Semigroups of Linear Operators and Applications to Partial Differential Equations (Applied Mathematical Sciences), vol. 44. Springer, New York (1983)

22. Yan, Z., Yan, X.: Existence of solutions for a impulsive nonlocal stochastic functional integrodifferential inclusion in Hilbert spaces. Zeitschrift furangewandte Mathematik und Physik 64(3), 573-590 (2013)

23. Debbouche, A., Torres, D.F.M.: Approximate controllability of fractional nonlocal delay semilinear systems in Hilbert spaces. Int. J. Control 86, 1577-1585 (2013) 